\title{
Estudio de la flexibilidad en la burocracia de la gestión pública en las universidades ecuatorianas
}

\section{Study of flexibility in the bureaucracy of public management in ecuadorian universities}

\author{
MONTENEGRO, Daniela M. ${ }^{1}$ \\ PÉREZ, Osmany ${ }^{2}$

\section{Resumen} \\ La burocracia constituye un elemento indispensable para el funcionamiento de las instituciones dado \\ que permite el cumplimiento de objetivos en función de la forma de gestionar. Su aplicación rígida, \\ conduce a la no aplicación de técnicas-estrategias que mejoren los resultados. El objetivo es analizar la \\ flexibilidad en la burocracia de la gestión pública en las universidades ecuatorianas para la toma de \\ decisiones, empleándose un enfoque cualitativo, sustentado en el método análisis-síntesis. Asimismo, \\ el tipo de estudio es descriptivo-explicativo. \\ Palabras clave: burocracia, flexibidad, universidades ecuatorianas
}

\begin{abstract}
Bureaucracy is an essential element for the functioning of institutions, since it allows the fulfillment of objectives depending on the way of managing. Its rigid application leads to the non-application of techniques-strategies that improve results. The objective is to analyze the flexibility in the bureaucracy of public management in ecuadorian universities for decision-making, using a qualitative approach, based on the analysis-synthesis method. Likewise, the type of study is descriptive-explanatory.

Key words: bureaucracy, flexibility, ecuadorian universities
\end{abstract}

\section{Introducción}

En el Ecuador, la gestión pública de las universidades ecuatorianas constantemente ha sido blanco de críticas y desaprobación ciudadana, debido a que se ha desvirtuado su razón de ser incumpliéndose sus objetivos institucionales, lo que podría estar asociado a una rígida e inamovible burocracia universitaria, incapaz de actualizarse para satisfacer las necesidades de cada época, mucho menos para desarrollar con eficiencia, eficacia y efectividad los procesos sustantivos de la academia, la investigación, la vinculación con la sociedad y la gestión.

En tal sentido, el presente artículo enfoca tres momentos: 1 . Análisis de la teoría de la burocracia universitaria y el papel de la flexibilidad en la gestión universitaria. 2. Descripción de la metodología escogida. 3. Discusión de

\footnotetext{
${ }^{1}$ Estudiante de la Mestría en Derecho, mención Gestión Pública. Pontificia Universidad Católica. Ecuador sede Ambato. Correo electrónico de contacto: dannymmon@hotmail.com

${ }^{2}$ Coordinador de Investigacion. Pontificia Universidad Católica. Ecuador sede Ambato. Correo electrónico de contacto: operez@pucesa.edu.ec
} 
los resultados obtenidos respecto de los siguientes indicadores: pertinencia de la oferta académica-profesional universitaria y empleabilidad, inversión en investigación científica y niveles de producción de las universidades ecuatorianas y vinculación con la sociedad y satisfacción de demandas ciudadanas, para finalmente emitirse varias conclusiones que orienten al lector sobre cómo la flexibilización de la burocracia universitaria puede potencializar el claustro del saber.

\subsection{Teoría de la burocracia universitaria y el papel de la flexibilidad en la gestión universitaria}

El estudio de la burocracia se enfoca en una forma de organización humana tan antigua como el hombre, incipiente en su génesis como una práctica empírica, pero robusta como teoría a partir del surgimiento del Estado moderno, de la escuela estructuralista y de la democracia de masas alrededor de los años 40 . Al respecto, existen diferentes pensadores y teorías construidas que resaltan criterios esenciales relacionadas con la burocracia en sentido general. En la literatura sobre este tema se destaca el papel de Weber (2001), como uno de sus principales defensores, quien consciente de las pequeñas limitaciones, la concibe como el modelo de intervención administrativo y estatal para la consecución de objetivos organizacionales a través de la sectorización, jerarquización, meritocracia, rigidez normativa y procesos definidos, por referir algunas características generales.

Relacionado a lo expuesto, se suman varios criterios coincidentes con el modelo de una burocracia rígida, como el de Fayol (1987). En su trabajo considera que, la operación administrativa es la más relevante para la gobernanza de una organización, destacándose que ésta, se centra en: capacidad de mando o autoridad, control, división del trabajo, empleo de procesos establecidos, disciplina y en la formalidad. En este mismo sentido, Taylor (1987) confirma que, se debe seleccionar a los mejores hombres para el trabajo, instruirlos eficientemente, supervisarlos, emplear métodos formales y procedimientos regulados con una estandarización de herramientas e instrumentos. Asimismo, Mises (2005) señala que, la relación Estado-funcionario, debe ser burocrática por excelencia, manteniéndose un régimen autoritario e inamovible por seguridad del ciudadano.

A su vez, en la literatura consultada, se aprecia que existen detractores de la teoría de la burocracia por considerarla idealista, caduca e incapaz de satisfacer a través de un sistema rígido, formal, limitado y general, las necesidades sociales, dado que, la relacionan de manera peyorativa con la organización pública, provista de corrupción, ineficiencia del servicio detrás de un escritorio y el papeleo excesivo, entre otros aspectos negativos, que impiden aplicar soluciones rápidas, específicas y adecuadas para lograr los objetivos de la organización, proponiéndose por su parte, la idea de una burocracia (organización) informal, adaptativa, abierta, en donde se dé importancia al comportamiento humano para prever amenazas (Merton, 1949; Olsen, 2005; Chiavenato, 2007; Torres, 2014). Con lo cual, las expectativas y necesidades reales y actuales del quehacer organizacional en favor del interés general, debe cambiar para que los procesos sean más ágiles y satisfagan las necesidades de las personas que requieren un determinado servicio en las instituciones universitarias.

Con respecto a lo planteado, están los pensadores que prefieren combinar ambos enfoques planteados como estrategia organizativa, ya que, si bien reconocen que, el carácter formal, reglado y organizado de la burocracia, evita conductas abusivas, arbitrarias y desordenadas de los funcionarios, y, legitima las decisiones, también consideran que, preservarla inamovible y desactualizada, resta valor al servicio, pues crea una brecha entre el operador y el ciudadano, limita la iniciativa propia y, mediante la práctica de actividades rituales, reduce la adaptación al cambio, especialmente, en las instituciones públicas caracterizadas por su rigidez (Crozier, 1969; Petrella, 2007; Zamudio y Arellano, 2011).

En tal sentido, analizar a la burocracia desde los extremos (sea éste positivo o negativo), podría impedir su valoración con objetividad, ya que, si bien presenta falencias y debilidades de obligatoria e inmediata atención, 
desconocerla y alejarla de la organización por considerarla desactualizada, ineficiente y poco práctica, podría constituir un error, puesto que, como señala Román (1976), sería privar a las organizaciones de un sistema estable de relaciones y procesos coordinados que permiten el funcionamiento constante de la gestión pública pese a sus imperfecciones.

Es así que, se concuerda con la idea de rescatar los aspectos relevantes de sus seguidores y detractores, para amalgamarlos en una burocracia moderna y flexible como estrategia organizacional que permita adoptar medidas pertinentes y actuales ante los requerimientos de la época, especialmente, con enfoque en las organizaciones públicas, y, en este caso, en las universidades, en donde el carácter formal, racional y de control se manifiestan permanentemente y en ocasiones de manera excesiva, desvirtuándose la razón de ser del claustro del saber y alejándolas del logro de sus objetivos estratégicos.

En tal contexto, se propone flexibilizar a la burocracia en la gestión pública de las universidades para mejorar y/o perfeccionar sus procesos y medios de atención a los usuarios internos y externos, dado el papel que este tipo de institución desempeña, por cuanto, a través de ella, se puede lograr el equilibrio dinámico entre la satisfacción de necesidades y el progreso social. De esta manera, se confirma lo expresado por Saldaña (2017) cuando refiere que "la Sociedad y la Universidad tienen una relación directa, bilateral e indisoluble vinculada al desarrollo histórico, social, político, económico y cultural de las naciones" (p. 224), cuya adecuada gestión del conocimiento, permitiría aprovechar un capital intangible en sus diferentes expresiones para una mejor calidad de vida. Esta perspectiva, deja entrever la responsabilidad de la universidad con los aspectos sociales, por lo que, la burocracia no debe entorpecer o limitar la rápida respuesta que necesita la sociedad en su conjunto para dar solución a sus disímiles problemas.

Con lo expresado, se corrobora que son variadas las teorías sobre el origen de las universidades. Una de estas teorías la expone Chuaqui (2002), donde refiere que, la palabra universitas fue creada probablemente por Cicerón para referirse a un universo reunido en un todo, vocablo que, al combinarse con las palabras magistrorum et.scholarium, harían alusión a la universidad o comunidad de maestros y alumnos (Mora, 2018). Es por ello que, con la aparición de estos centros de educación superior, se piensa que, nació la burocracia dado su funcionamiento y forma de prestar servicios, sin delimitar si éstas son privadas o públicas.

Sobre el tema, y sin el afán de restarle valor al origen de las universidades, ni polemizar sobre sus distintas transformaciones y funciones a lo largo de la historia, el enfoque del estudio profundiza el término de burocracia en sus procesos sustantivos: docencia, investigación, vinculación con la sociedad y gestión, los cuales se describen en la Ley Orgánica Reformatoria a la Ley Orgánica de Educación Superior (LOR. LOES, 2018, Arts. 10, 19). De esta manera, estos procesos sustantivos, engloban la razón de ser de las universidades para lograr formar, educar capacitar a las personas de manera holística para que estén preparadas para vivir, servir y trabajar en una sociedad cambiante y globalizada; por lo cual, es importante estudiarlos para poder revelar causas y/o factores que en ocasiones la alejan del cumplimiento de sus objetivos, restándole eficiencia, eficacia y efectividad al servicio que ofrecen al usuario interno y externo.

Sobre lo expresado, la Constitución de la República del Ecuador (CRE, 2008) resalta en sus artículos 343 y 344 , que, el sistema nacional de educación funcionará de manera flexible y dinámica para satisfacer al sujeto que aprende a través de políticas, programas y acciones adecuadas que fortalezcan el sistema nacional de educación. Con lo cual, la burocracia no debe entorpecer estos lineamientos que garantizan un nivel de satisfacción y alto desarrollo para la sociedad, al entregarle profesionales que respondan a sus problemas reales.

Es por ello que, esta investigación propone emplear una burocracia flexible para el desarrollo de la gestión pública de las universidades, esto con el fin, de enfocarse en la satisfacción de las necesidades del usuario final, 
así como, en el logro de los objetivos y el cumplimiento de la visión, misión y valores de la institución. Por lo tanto, los esfuerzos para una adecuada gestión universitaria se han de concentrar en optimizar el conjunto de servicios que prestan las personas, restándole importancia al modo o forma, sin dejarlo de lado, dado el carácter normativo y reglado de la burocracia, para concentrarse en el fin y en la ejecución de acciones que potencialicen el adecuado uso de recursos técnicos, financieros y humanos como transformadores de la persona y la sociedad (Botero, 2009; Larraín y Waissbluth, 2018).

En tal contexto, en los últimos años, el Estado ecuatoriano ha impulsado y orientado sus esfuerzos en el logro de una transformación real de sus universidades, sobre la base de una educación superior de calidad con niveles de excelencia. Sin embargo, lo hecho aún resulta insuficiente, ya que, en este escenario, no son pocas las áreas y ámbitos de cambios a los que la universidad tiene que hacer frente (Jarvis, 2006), resaltándose la importancia de trabajar en el perfil del estudiante en la formación de conocimientos, en el papel que desempeña el profesor y en sus prácticas docentes, pero además, en el uso de técnicas y estrategias de desarrollo y liderazgo organizacional de gestión empresarial y de talento humano para el cumplimiento de objetivos, panorama que describe a una Universidad Corporativa (UC).

Razón por la cual, Garzón (2019) define a la UC, como aquella Institución que debe adaptar su acción a las necesidades de las nuevas generaciones, debe evolucionar en sus propias prácticas para corresponder mejor a las expectativas de nuevos públicos, debe desarrollar nuevas formas de aprendizaje y hacer evolucionar el papel del formador en consecuencia. Corroborándose con ello, la necesidad de servir a sus usuarios en el menor tiempo posible, con una burocracia flexible y comprensible dado los procesos que se ejecutan en cada una de las áreas funcionales de este tipo de organización, pues de alguna manera, los procesos de un departamento dependen del otro, sean estos administrativos o académicos, y todos contribuyen a la formación general e integral de los profesionales.

Es así que, a partir de la perspectiva planteada, es posible comprender los beneficios de la flexibilidad de la burocracia para la adecuada gestión universitaria, transmutando, a medida de lo posible, el aspecto formal por lo funcional, con miras a cumplir lo dispuesto en el Art. 107 de la Ley Orgánica de Educación Superior (LOES, 2010), respecto al principio de pertinencia, que determina: la educación superior debe responder a las expectativas y necesidades de la sociedad, a la planificación nacional, al régimen de desarrollo, a la prospectiva de desarrollo científico, humanístico y tecnológico mundial, y a la diversidad cultural, articulándose su oferta docente, de investigación y actividades de vinculación con la sociedad a la demanda académica, a las necesidades de desarrollo local, regional y nacional.

\section{Metodología}

La metodología que se emplea en esta investigación es de tipo cualitativa, dado que, el objetivo es analizar la flexibilidad en la burocracia de la gestión pública en las universidades ecuatorianas, ya que, influye en la toma de decisiones de los procesos sustantivos y en sus niveles de eficiencia, eficacia y efectividad; considerándose para el efecto, tres indicadores: docencia desde la pertinencia académica, investigación (niveles de producción científica y las trabas para su desarrollo); y, vinculación con la sociedad. Asimismo, el tipo de investigación es descriptiva-explicativa; a su vez, se desarrolla el método de análisis-síntesis como es característico en estos tipos de estudios.

De la misma manera, en el aspecto metodológico se coincide con algunos autores: Strauss y Corbin, (2002); Hernandez, Fernández y Baptista (2006) en la necesidad de la utilización del método de análisis documental, pues, permitió profundizar en la literatura y en el marco legal ecuatoriano, con el propósito de conocer las 
competencias y potestad estatal de las universidades con respecto a la burocracia y la data de portales abiertos, de manera, que se pueda contrarrestar los efectos de la realidad ecuatoriana.

\section{Resultados y discusión}

Dado lo expuesto hasta el momento en el estudio sobre la burocracia pública universitaria, se puede confirmar que, consiste en la administración estatal que realiza una institución de educación superior para la consecución de sus objetivos organizacionales, a partir del análisis de indicadores relacionados con la gestión de los procesos sustantivos, entre los que destacan: docencia, investigación y vinculación con la sociedad, con la finalidad, de comprender si la actuación de la burocracia ha repercutido de forma favorable o no en su eficiencia, eficacia y efectividad.

\subsection{Pertinencia de la oferta académica-profesional universitaria y empleabilidad}

Sobre el tema que se aborda, el marco legal ecuatoriano reconoce que las universidades cuentan con la libertad para elaborar sus planes y programas de estudio encaminados a la formación superior académica-profesional de los jóvenes como actores estratégicos del desarrollo del país. En este sentido, su visión está orientada a formar profesionales capaces de resolver los problemas económico-sociales de la nación, dotándolos de una oferta académica actualizada y pertinente acorde a los requerimientos del desarrollo nacional (CRE, 2008, Arts. 39, 350; LOES, 2010, Art. 18, lit. c, Art. 107; LOR. LOES, 2018, Art. 8, lit. e).

Por lo tanto, "Identificar con antelación lo que el mercado requiere es condición necesaria para construir un sistema de educación y capacitación que facilite trayectorias de aprendizaje y laborales exitosas" (Banco Interamericano de Desarrollo [BID], 2019), considerándose clave, involucrar al sector productivo para identificar sus necesidades y tendencias, dado que, en el Ecuador de cada 100 plazas de trabajo, 92 fueron generadas por el sector privado y 8 por el sector público (Instituto Nacional de Estadísticas y Censos, INEC, 2021, p. 24). Con lo cual, y sustentado en el criterio expuesto, la oferta académica como proceso sustantivo debe dotar a los futuros profesionales de herramientas que les permitan ser jóvenes integrales en su formación y que logren convertirse en profesionales que transformen los problemas en soluciones sociales, a partir de los valores adquiridos en las instituciones de educación superior, donde prevalezca lo humanista como: responsabilidad, ética, laboriosidad, productividad, entre otros, que contribuyan a enfrentar y eliminar las barreras burocráticas que entorpecen el desarrollo integral de las universidades.

En tal sentido, este estudio puede ser considerado en todas las universidades ecuatorianas, dado que, aún el esfuerzo universitario no se ha concentrado en potencializar los procesos sustantivos mencionados en esta investigación. Es por ello, que se continúa promoviendo ofertas académicas con carreras tradicionales saturadas, por ejemplo, Administración, una carrera sobre demandada y de baja competitividad a nivel nacional e internacional, lo cual, confirma lo expuesto por el BID, siendo una de las profesiones que está en declive, y que podría llegar a ser automatizada y reemplazada por las nuevas tecnologías (BID, 2019; 2020).

Por otro lado, el BID (2019) señala que, existen otras profesiones con gran demanda y futuro en América Latina, las cuales están relacionadas al área digital y tecnológica, que a pesar de reportar un incremento considerable del 1884\% en el número de matriculados en el Ecuador (de 494 en el año 2015 a 9311 en el año 2018), específicamente, en el área de Tecnologías de la Información y Comunicación (TIC) (SENESCYT, 2019), constituyen cifras insuficientes, dada la escasez de capital humano relevante para ciertas industrias que se consolidan en el mercado nacional e internacional.

Con la información proporcionada por la SENESCYT (2019), concuerda el BID (2020), al confirmar que, existe la necesidad, el incentivo y el interés por alcanzar un alto desarrollo de habilidades en el campo de la informática, 
destacándose temas relacionados con: uso de la tecnología, big data, automatización, empleo de robots, inteligencia artificial, adopción de trabajo remoto por imposibilidad de proximidad física, uso de plataformas digitales, entre otras, ya que, en la actualidad hay un creciente porcentaje de personas con estudios superiores que no pueden encontrar empleo como profesional y terminan empleándose en otras ocupaciones no afines a su formación, pero que, exigen de estos conocimientos.

Según lo expuesto, la iniciativa de empresas privadas no se ha quedado a la espera de cuándo se va a lograr un cambio de las ofertas de carreras en las diversas universidades $y$, como contrapartida, en función de sus necesidades de contar con talento humano preparado y mejor formado, se ha propuesto capacitar a su personal en diferentes áreas del conocimiento, mediante ofertas académicas acordes a las necesidades y problemas del país, revelándose la necesidad de flexibilizar la burocracia universitaria. Dado esta perspectiva, por ejemplo, la Escuela de Negocios de la Cámara de Comercio de Quito (CCQ, 2021), conjuntamente con la Escuela Europea de Dirección y Empresa de la European Business School (EUDE), ofertan maestrías, especializaciones, cursos, diplomados y talleres en áreas relacionadas, con: marketing digital, gestión digital de la empresa, social media, analítica web, logística - ecommerce, estrategia y transformación digital, entre otras, asemejándose a una UC.

De la misma manera, el BID (2019) advierte que, en la actualidad resultan insuficientes únicamente los conocimientos técnicos, puesto que, el profesional también requiere de habilidades transversales y blandas, como las que se detallan en el cuadro 1. Sin embargo, éstas son impulsadas de forma limitada por los pensum académicos universitarios tradicionales, pues, escasamente se imparten módulos relacionados al liderazgo, sin percibir que, las ocupaciones o profesiones rutinarias desarrolladas de modo poco flexible, son más propensas a ser reemplazadas. Así, el $50 \%$ de las tareas que se realizan en las organizaciones por el $80 \%$ de la fuerza laboral mundial podrían ser sustituidas por las nuevas tecnologías. Por ejemplo, en el Ecuador, el $69 \%$ de trabajadores pueden ser reemplazados por robots, dejándose entre ver con ello, que puede exitir en el futuro un elevado nivel de desempleo y pobreza en la nación (BID, 2019, 2020).

\section{Cuadro 1}

Habilidades transversales/blandas

demandadas en América Latina

\begin{tabular}{|l|}
\hline Capacidad de resolución de problemas \\
\hline Capacidad de trabajo en equipo \\
\hline Pensamiento creativo y crítico \\
\hline Capacidad de comunicación e interacción \\
\hline Adaptabilidad al cambio \\
\hline Flexibilidad \\
\hline Capacidad de aprender \\
\hline Liderazgo \\
\hline
\end{tabular}

Fuente: Banco Interamericano de Desarrollo. ¿Cuáles son las ocupaciones y las habilidades emergentes más demandadas en la región?, 2019

Elaboración propia.

Como se puede apreciar en el cuadro anterior, ya existe una identificación de habilidades necesarias a desarrollar desde la academia y la investigación durante todo el desarrollo y formación de los nuevos profesionales. De esta manera, las mallas de estudios deben considerar en las diversas carreras esta percepción de los autores y esta necesidad para las empresas. 


\subsection{Inversión en investigación científica y niveles de producción de las universidades ecuatorianas}

La investigación es una actividad innata del hombre debido a su curiosidad e ingenio por descubrir el mundo y desarrollarse en él. Su importancia se asocia al progreso y evolución del individuo y de las naciones. Así lo prevé el Estado ecuatoriano en la CRE (2008) cuando reconoce la obligación de promover en la sociedad la generación, adaptación, difusión, producción-transmisión del conocimiento y del fomento de la investigación científicatecnológica para el impulso de la producción nacional y el fortalecimiento de la economía. Para el efecto, se deben destinar recursos suficientes del presupuesto nacional entre sus diferentes actores, destacándose entre ellos, las universidades como las principales productoras del saber (Arts. 385-388), pero además, se deben promover políticas públicas de fomento y desarrollo de la investigación, la tecnología, la ciencia y la innovación en esta área estratégica.

Sobre el tema, respecto a los recursos, según se describe en el gráfico 1, la inversión que el país ha realizado en investigación es mínima, alcanzándose al año 2014 apenas el 0,44 \% del Producto Interno Bruto (PIB), según datos del Banco Mundial (2014), produciéndose un ligero incremento al cabo de seis años para el $2020(0,47 \%)$ según datos de la SENESCYT (2020), que comparándose con la inversión que realizaron países del primer mundo, denota gran retraso. Así, por ejemplo, el porcentaje del PIB invertido en el año 2014 por los países objeto de análisis corroboran resultados muy superiores, en este sentido: Israel con 4,17\%; Japón con 3,40\%; Finlandia con 3,17\%; Alemania con 2,87\%; Estados Unidos con 2,72\%; y, China con 2,03\%, cuyas prósperas economías son el resultado de una adecuada inversión en investigación para la transformación del conocimiento como fuente generadora de valor, riqueza y desarrollo en sus naciones.

Por otro lado, está Latinoamérica encabezada por Brasil con 1,27\%; Argentina 0,59\%, superando a: Chile con 0,38\%; Uruguay; Venezuela con $0,34 \%$; y, Colombia con $0,31 \%$, países con economías emergentes cuya carencia investigativa repercute directamente en su desarrollo, lo que, a criterio de Santa y Herrero Solano citados por Araujo-Bilmonte, Huertas-Tulcanaza y Párraga-Stead, (2020) es el resultado de la realidad social, económica, política y cultural de la región, caracterizada por la corrupción, pobreza, hambre, insalubridad, violencia, entre otros aspectos, lo cual, evita que existan temas de tanta envergadura y que ocupen espacios en las agendas políticas o se los tome con la seriedad que merece.

Gráfico 1

Gasto en investigación y desarrollo (\% del PIB 2014)

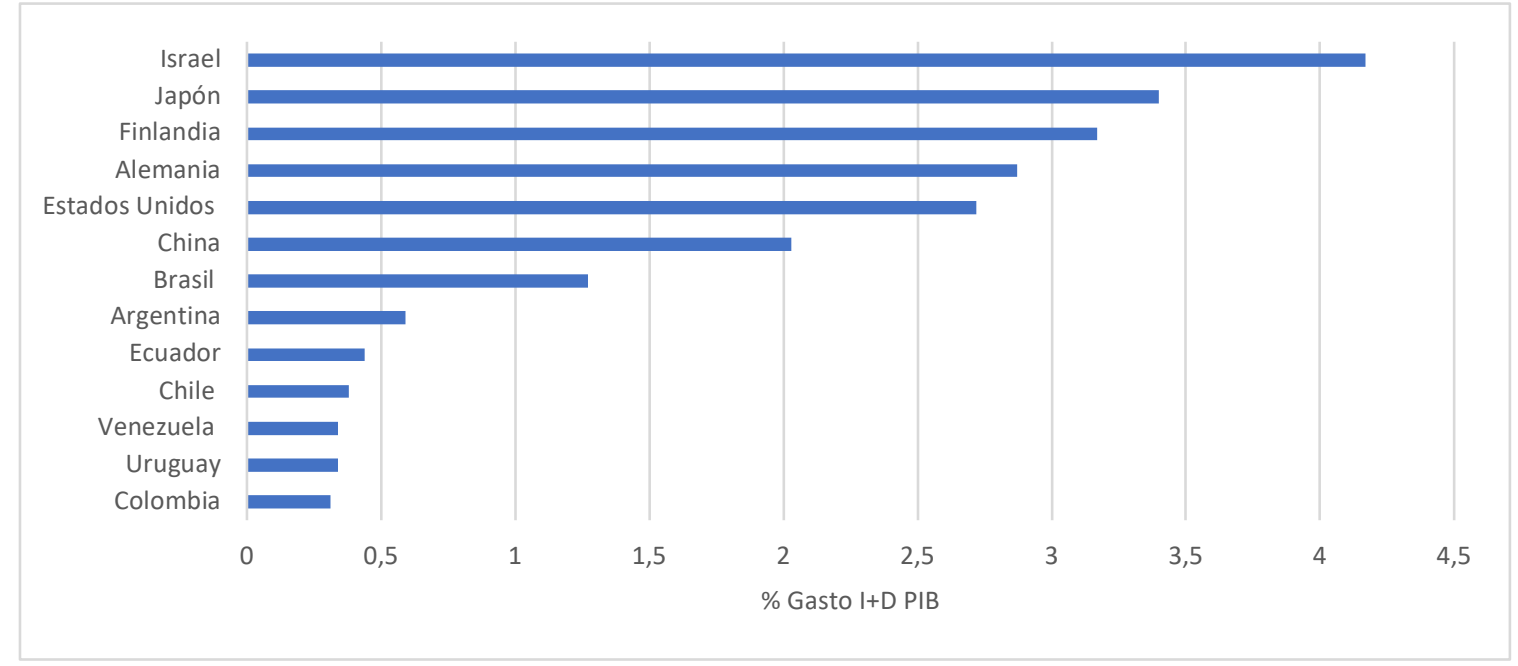

Fuente: Banco Mundial tomado de Instituto de Estadística de la Organización de las Naciones Unidas para la Educación, la Ciencia y la Cultura (UNESCO). 
Adicionalmente, en el Ecuador a partir del año 2008 con la promulgación de la CRE y el cambio de la matriz productiva con enfoque en la reestructuración del sistema de educación superior para fortalecer el conocimiento y el talento humano (Secretaría Nacional de Planificación y Desarrollo, 2012), se han expedido políticas públicas y bases normativas para la promoción, desarrollo y fortalecimiento de la ciencia y la investigación (Arts. 262, 298, $350,351)$, entre las que se destacan: la creación de Yachay Tech como la universidad para la investigación y la innovación del país, el programa Prometeo cuyo fin es atraer científicos y académicos extranjeros de alto nivel para el desarrollo de investigación local, el Banco de ideas de la SENESCYT para el impulso práctico de la innovación y el conocimiento, las becas de la SENESCYT para estudios exclusivos de posgrado, la promulgación del Código Orgánico de la Economía Social de los Conocimientos, la Creatividad y la Innovación [INGENIOS] como norma regulatoria del Sistema Nacional de Ciencia, Tecnología, Innovación y Saberes Ancestrales y su vinculación con la educación (Ingenios, 2016; Vizioli, 2016; Senescyt, 2020).

Por su parte, estas iniciativas están enfocadas en potencializar seis ejes de acción en el campo de la investigación y la ciencia para el desarrollo productivo del país con base en el conocimiento, mismos que se enumeran a continuación en el cuadro 2 y cuya profundización constituirá motivo de análisis de estudios posteriores, siendo referenciales para esta investigación.

\section{Cuadro 2}

Seis ejes de acción para el desarrollo de la Investigación

como política pública en el Ecuador

Desarrollar los recursos humanos en ciencia y tecnología

Impulsar la generación y potenciación de la investigación científica

Desarrollar tecnología e innovación

Estructurar el sistema de ciencia y tecnología

Impulsar la creación de academias de ciencia, comités de ética e incorporar los resultados de la investigación al aparato productivo para mejorar el nivel de vida

Incorporar TIC's para difundir el conocimiento generado.

Fuente: La innovación en la política pública de ciencia y tecnología ecuatoriana.

Yachay: ¿Un cluster de innovación? (Viziol, 2016).

Sobre lo expuesto, y pese a la voluntad política para impulsar los ejes para el desarrollo de la investigación en el Ecuador, los esfuerzos realizados aún resultan insuficientes e impiden alcanzar niveles óptimos en el campo de la ciencia para el impulso productivo del país, ya que, a criterio de Escobar (2016) aún no se supera el enfoque discursivo por acciones concretas que maximicen los beneficios de la inversión pública, dado que, existe claridad de los principios teóricos pero carencia de procedimientos pertinentes, eficientes, eficaces y efectivos. Así, por ejemplo, en cuanto a la política de becas de la Senescyt existe desvinculación entre el programa específico elegido por el becario y las necesidades del país, ya que "el $44 \%$ de los beneficiarios estudian temas no relacionados directamente al cambio de la matriz productiva" (Escobar, 2016, p. 43), imposibilitándose el uso del talento humano en áreas estratégicas.

En otro sentido, para Tamayo, citado por Ortega y Guerrero (2021) las limitaciones para el desarrollo de la ciencia podrían estar relacionadas al modo unilateral del Estado para la construcción de la política pública y a su falta de legitimación por parte de los destinatarios, ya que se ha excluido a los principales actores de la investigación del proceso de identificación y definición de los problemas del país, de la formulación, adopción e implantación de las alternativas y de la evaluación de los resultados obtenidos, limitándolos únicamente a cumplir directrices impuestas por organismos de control que en ocasiones, además de ser poco específicas, no pueden ser aplicadas por la carencia de recursos y/o por el modelo de burocracia empleado para el desarrollo de su gestión. 
Es así que, en el escenario planteado en este estudio, para la universidad ecuatoriana como actor estratégico para el desarrollo de la investigación formativa de tercer y cuarto nivel (Codificación Reglamento de Régimen Académico del Consejo de Educación Superior, RRA CES, 2020, Arts. 40, 42, 43), constituye un verdadero desafío adquirir, generar, transmitir y/o difundir el conocimiento científico, a partir de las limitaciones económicas que persisten en las instituciones de educación superior por los escasos presupuestos asignados y que tienden a la baja (Sentencia No. 9-20-IA/20, 2020), además, de los factores burocráticos que entorpecen y alejan a este proceso sustantivo de los niveles de eficiencia, eficacia y efectividad que la sociedad espera, como los que se describen en el cuadro 3 por referir algunos, haciéndose necesaria su inmediata flexibilización.

\section{Cuadro 3}

Principales problemas burocráticos para desarrollar investigación científica en las universidades públicas ecuatorianas

Escazas competencias investigativas de los docentes.

Insuficientes capacitaciones sobre metodología de la investigación y redacción científica.

Excesivas gestiones administrativas para conseguir financiamiento para proyectos.

Presentación exhaustiva de planificaciones, formatos, informes justificativos.

Extensos trámites para gestionar viajes a congresos, seminarios, ponencias para presentar las publicaciones, perfeccionamiento docente.

Inexistencia de grupos de investigación.

Ausencia, escasez u obsolescencia de laboratorios, equipos, instrumentos y/o reactivos.

Fuente: Estudio del ingreso de los bachilleres a la universidad: mecanismos y procesos de admisión (Analuiza, 2016); La investigación científica: una función universitaria a considerar en el contexto ecuatoriano (Dáher, Panunzio y Hernández, 2018). La burocracia universitaria (Muñoz, 2019).

Con respecto a lo expuesto, debe profundizarse en cómo ha respondido la burocracia universitaria. En este sentido, las escazas competencias de los docentes en el área de la investigación podría deberse a un inadecuado proceso de contratación del talento humano por parte de las universidades, dado que, las actividades docentes y de investigación entre otras, consisten en el diseño y elaboración de libros, dirección y tutorías de trabajos de titulación, diseño y desarrollo de proyectos de investigación básica y el uso pedagógico de la investigación para la enseñanza, participación en redes y programas de investigación, participación en congresos, seminarios de investigación (Reglamento de Carrera y Escalafón del Personal Académico del Sistema de Educación Superior, 2021, Arts. 6, 7), sin que, los perfiles seleccionados puedan satisfacer la demanda científica del país, pese a que un $65 \%$ de docentes cuenten con maestría y un $12 \%$ con doctorado, según reportes de la SENESCYT (2020) para el año 2018.

Asimismo, se suman a la carencia de conocimiento, un sinnúmero de trabas administrativas en la práctica, que ralentizan y en ocasiones anulan el desarrollo de la investigación, entre las que se destacan: la inexistencia de grupos de investigación, excesivas gestiones administrativas que los docentes e investigadores deben realizar para conseguir financiamiento para sus proyectos, presentación exhaustiva de planificaciones, formatos, informes justificativos, extensos trámites para gestionar viajes a congresos, seminarios, ponencias, para presentar publicaciones o para perfeccionamiento docente, todo aquello previa aprobación por parte de instancias académico-administrativas, por referir algunas.

Por ejemplo, en una de las universidades públicas del centro del país seleccionada al azar como referencia, el tiempo de tramitación de una petición docente de comisión de servicios y asignación de beca para el desarrollo de estudios doctorales, tarda alrededor de cuatro meses desde la solicitud hasta su aprobación, dado que, durante el proceso se ha solicitado a seis instancias académico-administrativas varios informes de sustento y procedencia, que si bien, por un lado buscan proteger la legalidad de la asignación de recursos y beneficios, por 
otro, desmotivan a los docentes a generar competencias y conocimiento en el campo de la investigación, pudiéndose manejar este aspecto de un modo menos rígido para facilitar el fortalecimiento de este proceso sustantivo (Resolución 1222-CU-P-2020, 2020).

Asimismo, se evidencia una falencia burocrática universitaria en cuanto a la capacitación docente sobre metodología de la investigación y redacción científica, cuya repercusión se evidencia en la calidad y cantidad de producción científica, en este sentido y a modo de referencia, se observa en la base de datos Web of Science (WOS) que almacena información de importantes bases de datos de carácter internacional que durante el año 2019 el Ecuador tuvo 4332 registros de producción científica, que comparado con 20 países latinoamericanos, caracterizados por su incipiente impacto en la investigación, alcanza el séptimo lugar (Araujo-Bilmonte, HuertasTulcanaza y Párraga-Stead, 2020), evidenciándose así, un retraso dentro de la región en este indicador, siendo ocasionado en la generalidad de los casos por situaciones burocráticas innecesarias.

A ello se suma, el tema de la calidad del impacto de la producción científica ecuatoriana, que en su gran mayoría es de alcance nacional o regional más no de alto impacto (Barros y Turpo, 2020), debiendo los investigadores ecuatorianos adherirse a investigaciones extranjeras, especialmente, europeas o estadounidenses para tener impacto internacional de mayor nivel (Castillo y Powell, 2019), mediante importantes redes de investigación, pese a que según el Modelo de Evaluación Externa de Universidades y Escuelas Politécnicas, los estándares están dirigidos a analizar la planificación y ejecución de la producción académica y científica, así como los resultados reflejados (Consejo de Aseguramiento de la Calidad de la Educación Superior [CACES], 2019).

Finalmente, y ante la incompetente gestión de diversas universidades, cabe preguntarse cómo se puede impulsar y fortalecer la investigación universitaria con ausencia, escasez u obsolescencia de laboratorios, equipos, instrumentos y/o reactivos, que limitan las verdaderas potencialidades de la formación de los futuros profesionales del país. Pues, resulta ser una tarea difícil lograr mejores resultados sin la presencia de recursos suficientes, ya sea, por falta de financiamiento, desinterés de las autoridades de las instituciones o por elementos excesivamente burocráticos que crean barreras insostenibles para el desarrollo, por ejemplo: limitada adquisición de equipos y reactivos para las investigaciones, laboratorios con bajo nivel tecnológico para las investigaciones debido al costo de adquisición, tardanza en las compras de insumos para el desarrollo de investigaciones importantes en ramas esenciales de la economía de un país, baja competitividad internacional en la formación de doctores con competencias investigativas, y limitada calidad en la formación de profesionales de la universidad ecuatoriana producto de los pensum tradicionales de estudios.

Todo lo expuesto, corrobora la necesidad de flexibilizar la burocracia universitaria y la adopción de estrategias que permitan aprovechar con eficiencia, eficacia y efectividad el $6 \%$ destinado del presupuesto para "ejecutar proyectos de investigación, adquirir infraestructura tecnológica, publicar textos pertinentes a las necesidades ecuatorianas en revistas indexadas, otorgar becas doctorales a sus profesores titulares y pago de patentes", derivadas de la producción científica al más alto nivel nacional e internacional (LOR. LOES 2018, Art. 28).

A su vez, es momento de aprovechar las exoneraciones de derechos aduaneros que tienen las universidades para la importación de artículos y materiales para la investigación científica, con la finalidad de equipar los laboratorios, adquirir mejores equipos tecnológicos, comprar insumos y reactivos necesarios para los diversos estudios (LOR. LOES 2018, Art. 30), mediante la aplicación de políticas pertinentes en la teoría y en la práctica y no tan burocráticas que fortalezcan a la academia, dotándoles con conocimientos técnicos y científicos específicos que coadyuven a resultados superiores a los alcanzados hasta la fecha, segunda década del siglo XXI. 


\subsection{Vinculación con la sociedad y satisfacción de demandas ciudadanas}

Otra de las variables del estudio es la Vinculación con la Sociedad, que es considerada una función sustantiva de la universidad ecuatoriana, cuyo enfoque es participativo y solidario dentro de una sociedad que aprende y comprende la inclusión y contribución social en el desarrollo permanente local y nacional, en el interés público y en la iniciativa comunitaria para crear y trabajar con miras a solucionar problemas sociales, ambientales y productivos, con especial atención en los grupos vulnerables. Asimismo, ésta guarda estrecha relación con la pertinencia académica y la investigación. Su desarrollo depende de la participación de docentes, investigadores y estudiantes, para quienes, además, constituye un requisito para la titulación de tercer nivel (CRE, 2008, Arts. 26, 27, 28; LOR. LOES, 2018, Art. 6, 10, 19, 67, 68, 91; RRA. CES, 2020, Art. 4). De esta manera, tiene ciertas características que se describen en el cuadro 4.

El desarrollo de las variables planteadas y su cumplimiento, depende directamente de la gestión pública universitaria. Dado que, con su análisis se pueden proyectar estrategias y acciones planificadas, eficientes, eficaces y efectivas que permitan adecuar el conocimiento teórico de la profesión a la práctica laboral, pero principalmente a la investigación, mediante la adquisición de herramientas suficientes para identificar las necesidades, el campo de acción, los beneficiarios, los recursos o el talento humano, así como, para ejecutar, difundir y evaluar "actividades que garantizan la participación efectiva en la sociedad y la responsabilidad social de las instituciones del Sistema de Educación Superior" (RRA. CES, 2020, Art. 43, 50), de ahí, su estrecha relación e interdependencia entre todas las funciones sustantivas, y la necesidad de fortalecerlas en su conjunto. Empleándose para ello, prácticas burocráticas flexibles que garanticen el cumplimiento pleno de sus fines y objetivos.

\section{Cuadro 4}

Características de la vinculación con la sociedad según el marco legal ecuatoriano

\begin{tabular}{|l|}
\hline Democratiza el conocimiento. \\
\hline Contribuye con la pertinencia del quehacer educativo. \\
\hline $\begin{array}{l}\text { Genera promoción y transferencia de conocimiento académico-científico-tecnológico entre docentes, } \\
\text { investigadores y estudiantes para la sociedad. }\end{array}$ \\
\hline $\begin{array}{l}\text { Forma integralmente a los estudiantes, combinando la teoría con la práctica en los procesos de } \\
\text { enseñanza-aprendizaje. }\end{array}$ \\
\hline Promueve espacios de experiencia vivencial y reflexión crítica. \\
\hline Promueve la transformación social desde un enfoque de derechos, equidad y responsabilidad social. \\
\hline Garantiza la construcción de respuestas efectivas a las necesidades y desafíos del entorno. \\
\hline $\begin{array}{l}\text { Mejora la calidad de vida, el medio ambiente, el desarrollo productivo y la preservación, difusión y } \\
\text { enriquecimiento de las culturas y saberes. }\end{array}$ \\
\hline Promueve el desarrollo de la innovación social y la divulgación científica. \\
\hline
\end{tabular}

Fuente: RRA. CES, 2020 (Arts. 4, 50, 51).

En virtud de lo expuesto, la vinculación con la sociedad se caracteriza por la transmisión del conocimiento universitario hacia la sociedad para la solución de sus problemas y la construcción de un mejor estilo de vida para las comunidades, empleándose para el efecto, investigación, innovación y transmisión de conocimiento. Sin embargo, en las universidades ecuatorianas se debe realizar un trabajo profundo en esta área, dadas las debilidades que presenta (Ver cuadro 5), siendo una de las principales, el relacionar la vinculación únicamente con un enfoque social, como ocurre en el país, porque limita su campo de acción le resta valor e importancia a la innovación a partir de la investigación académica-científica. 
Cuadro 5

Debilidades de la Vinculación

con la Sociedad en Ecuador

\begin{tabular}{|l|}
\hline Enfoque netamente social. \\
\hline Escasa triangulación entre universidad, empresa y Estado. \\
\hline Inexistencia de proyectos a largo plazo. \\
\hline Falta de liderazgo y empoderamiento de los docentes asignados. \\
\hline Mallas curriculares desactualizadas e impertinentes. \\
\hline Escaza investigación e innovación universitaria. \\
\hline Excesiva burocracia académica-administrativa. \\
\hline
\end{tabular}

Fuente: Relevancia del proceso de vinculación con la sociedad en la formación de profesionales en instituciones de educación superior del Ecuador (López, Cedeño, Lescay y Mestre, 2016); La educación superior y su vinculación con la sociedad: referentes esenciales para un cambio (Barreno, M., Barreno, Z. y Olmedo, A., 2018); Las universidades y sus prácticas de vinculación con la sociedad (Rueda, Acosta y Cueva, 2020). Elaboración propia.

Al respecto, la falta de pertinencia académica influye de manera directa en la triangulación entre UniversidadEmpresa-Estado, dado que, la formación curricular estatal es incapaz de responder a las demandas de las organizaciones, al desarrollo productivo y a la reactivación económica del país, siendo necesario flexibilizar la burocracia universitaria en la academia para alcanzar niveles de calidad, que a su vez, faciliten el desarrollo de la vinculación con la sociedad. Asimismo, la inexistencia de proyectos a largo plazo, impide un desarrollo constante y sostenible en el tiempo de las estrategias universitarias para solucionar problemas sociales trascendentes (Viña, Sacoto y Landívar, 2019).

De igual forma, poco o nada se puede avanzar respecto a la vinculación si existe desmotivación y falta de empoderamiento por parte del personal docente responsable de desarrollar esta función con el estudiantado, ya que, en lugar de constituirse en un facilitador, en ocasiones puede ser un obstáculo en el proceso, ya sea por falta de conocimiento o vocación, siendo necesario cambiar paradigmas burocráticos desde su contratación, capacitación académica-científica y evaluación del desempeño, con miras a fortalecer esta valiosa función sustantiva. Es por ello, que es necesario potencializar la investigación con el propósito de generar un impacto social con el trabajo que desarrollan las universidades, y no el que en la actualidad se entrega y desdice del quehacer universitario dado sus bajos resultados y complicaciones propias (RRA. CES, 2020, Art. 43).

Así, por ejemplo, para tener una idea del estado en el que se encuentra la vinculación con la sociedad en las universidades ecuatorianas, se refiere que, el $95 \%$ de los estudiantes universitarios ecuatorianos y el $85.7 \%$ de los profesores reportan baja o muy baja participación en actividades para potencializar saberes ancestrales; el $97,8 \%$ de los estudiantes y el $92.8 \%$ de los profesores reflejan una baja y muy baja presentación de resultados científicos en congresos, seminarios y conferencias; el $94,3 \%$ de los estudiantes y el $78.6 \%$ de los profesores tienen una baja y muy baja publicación de resultados que muestren beneficios sociales de su actividad (López, Cedeño, Lescay y Mestre, 2016).

Asimismo, el $95,7 \%$ de los estudiantes y el $71.4 \%$ de los profesores reportan una baja y muy baja incorporación a proyectos de investigación e innovación con fines sociales; el $77.1 \%$ de los estudiantes y el $78,6 \%$ de los profesores consideran como baja y muy baja la colaboración en la revisión técnica documental para instituciones del Estado, por referir algunos parámetros evaluados, demostrándose que, las estrategias y acciones planificadas por las universidades para adecuar el conocimiento de la profesión a la práctica laboral y a la investigación, no han sido pertinentes y han restado calidad a esta función, haciéndose necesaria la flexibilización de la burocracia y la adopción de prácticas que prioricen la razón de ser de la universidad, como su impacto socio-económico para el progreso de la nación (López y otros, 2016; RRA. CES, 2020, Art. 50). 
Otro de los impedimentos que influyen en el adecuado desarrollo de la práctica vinculadora, es que existen procesos burocráticos que retardan y en ocasiones impiden la participación universitaria y el desarrollo de proyectos, investigaciones y/o publicaciones, por ejemplo, cuando se requiere transporte, materiales, equipos audiovisuales, suministros de oficina, financiamiento, por referir algunos. En este sentido, se deben seguir una gran cantidad de trámites ante instancias administrativas para justificar los egresos e inversiones, que si bien, por un lado, garantizan el adecuado manejo de recursos, por otro, restan interés y empatía por la extensión del tiempo para lograrlos. Bajo esta realidad, es posible comprender el motivo de la incapacidad de la vinculación con la sociedad en la actualidad, lo cual dificulta la transformación social en la práctica mediante proyectos, la difusión y la devolución de conocimientos académicos, científicos y artísticos, desde un enfoque de derechos, equidad y responsabilidad social (RRA. CES, 2020, Art. 51).

Asimismo, es evidente entender que, si la burocracia que dirige este proceso no adecua sus estrategias a satisfacer las debilidades expuestas, poco o nada podrán hacer las universidades en beneficio de la sociedad, pues carecen de: medios, herramientas, motivación, estrategias, personal, conocimientos e interés por trascender y renovar su entorno social, para servir y rescatar la imagen de la Universidad en la sociedad, siendo necesario para ello, realizar un giro en la dirección correcta para aprovechar la vinculación con la sociedad con la inteligencia del ser humano y con una práctica de la burocracia que no constituya barreras infranqueables.

\section{Conclusiones}

La burocracia de las universidades públicas ecuatorianas consiste en el empleo de modelos organizacionales para el desarrollo de las funciones sustantivas de la Docencia, Investigación, Vinculación con la Sociedad y la Gestión, la cual, es transversal a las demás y las viabiliza, guardándose estrecha relación e interdependencia entre todas ellas para la consecución de los objetivos institucionales, basándose en la implementación de políticas, normativas, jerarquías y estrategias definidas para la toma de decisiones proactivas. En este sentido, la burocracia tiene que ser flexible para adaptarse oportunamente a las cambiantes condiciones del mundo y a las demandas sociales de la época, por lo que, mantenerla rígida, desactualizada e inamovible podría constituir un grave error, capaz de repercutir de manera directa en los niveles de eficiencia, eficacia y efectividad, dado que, solo priorizaría la forma y la estructura institucional tradicional universitaria, por sobre su verdadera razón de ser, que es el servicio y la transmisión del conocimiento para el desarrollo y el progreso de la nación.

El estudio aplicó una metodología de tipo cualitativa, con el propósito de que el análisis de la situación actual de las universidades profundizara en la flexibilidad de la burocracia de la gestión pública y en la contribución de las ideas para la resolución de problemas, realizándose un análisis documental de la información relevante que reposa en libros, artículos de revistas, informes, marco legal ecuatoriano y la data de portales abiertos, concluyéndose que, aún existe gran cantidad de trabajo por ejecutar sobre el tema, dado los bajos niveles universitarios de calidad y pertinencia. En la docencia se corrobora la existencia de una deficiente gestión universitaria dada la escasez de profesionales para satisfacer las demandas de desarrollo productivo del país, régimen educativo tradicional, limitadas estrategias para adaptarse a los cambiantes desafíos de la sociedad y el mundo, ante lo cual, la empresa privada, debido a su necesidad de incorporar profesionales competentes y actualizados, han empezado a dar atisbos de llegar a constituirse en UC para solventar las carencias universitarias, haciéndose evidente la necesidad de flexibilzar la burocracia universitaria en esta función sustantiva para alcanzar niveles de pertinencia.

Asimismo, se aprecia una debilitada función de la investigación dados los resultados expuestos, que son consecuencia de la inexistencia de una directriz de política pública clara que oriente en la práctica la inversión del Estado para la satisfacción de las necesidades específicas del país, de la falta de legitimación de las mismas por parte de los actores que generan conocimiento como es el caso de las universidades lo que influye en su 
aplicación y pertinencia, de las limitaciones económicas, organizacionales, estatales y profesionales, pero especialmente, del empleo de una deficiente práctica burocrática, incapaz de impulsar a la investigación a nivel nacional e internacional, de potencializar la transferencia de conocimiento pertinente y relevante dejándose entrever que, en cuanto a la gestión universitaria, se tiene que replantear las estrategias y políticas adoptadas para que la universidad cumpla estándares de calidad y excelencia y realmente sirva a la sociedad.

A su vez, la burocracia universitaria ha impedido el desarrollo óptimo, pertinente y de calidad de la vinculación con la sociedad, dada la rigidez y obsolescencia de sus políticas y estrategias y a su incompetente gestión, lo que, la ha alejado del cumplimiento de sus fines, por ejemplo: traslado del conocimiento teórico a la práctica, transformación social, resolución de problemas, desarrollo de producción científica y la implementación de innovación para el desarrollo productivo del país, haciéndose evidente la necesidad de actualizarla y fortalecerla a través de nuevas prácticas organizacionales.

Finalmente, la burocracia universitaria es necesaria para el funcionamiento de las organizaciones educativas, por lo que, es imposible prescindirla, sin embargo, flexibilizarla adecuándola a las demandas del escenario ecuatoriano actual y priorizándose el cumplimiento de los fines y objetivos institucionales demandados por mandato constitucional y legal, es trascender la práctica administrativa y la gestión pública, con la finalidad de rescatar la legitimidad del claustro del saber en la sociedad.

\section{Referencias}

Analuiza, H. (2016). Estudio del ingreso de los bachilleres a la universidad: mecanismos y procesos de admisión. (Tesis de Maestría) Universidad Andina Simón Bolívar Sede Ecuador. Quito.

Araujo-Bilmonte, E., Huertas-Tulcanaza, L. y Párraga-Stead, K. (2020). Análisis de la producción científica del Ecuador a través de la plataforma Web of Science. Revista Cátedra, 3(2), 150-165.

Asamblea Nacional de la República del Ecuador (2008). Constitución de la República del Ecuador [CRE], R.O. No. 449.

Asamblea Nacional de la República del Ecuador (2010). Ley Orgánica de Educación Superior [LOES], R.O. No. 298.

Asamblea Nacional de la República del Ecuador (2016). Código Orgánico de la Economía Social de los conocimientos, creatividad e innovación [INGENIOS], R.O. No. 899.

Asamblea Nacional de la República del Ecuador (2018). Ley Orgánica Reformatoria a la Ley Orgánica de Educación Superior [LOR. LOES], R.O. No. 297.

Banco Interamericano de Desarrollo [BID] (2019). ¿Cuáles son las ocupaciones y las habilidades emergentes más demandadas en la región?. Recuperado de https://www.iadb.org/es/trabajo-ypensiones/ocupaciones-y-habilidades-emergentes-mas-demandadas-en-la-region

Banco Interamericano de Desarrollo [BID] (2020). El futuro del trabajo en América Latina y el Caribe.

Recuperado de https://publications.iadb.org/publications/spanish/document/El-futuro-del-trabajo-enAmerica-Latina-y-el-Caribe-Cual-es-el-impacto-de-la-automatizacion-en-el-empleo-y-los-salarios.pdf

Banco Mundial (2014). Gasto en investigación y desarrollo (\% del PIB). Recuperado de https://datos.bancomundial.org/indicator/GB.XPD.RSDV.GD.ZS 
Barreno, M., Barreno, Z., y Olmedo, A. (2018). La educación superior y su vinculación con la sociedad: referentes esenciales para un cambio. Universidad y Sociedad, 10(3), 40-45.

Barros, C., y Turpo, O. (2020). La formación en investigación y su incidencia en la producción científica del profesorado de educación de una universidad pública de Ecuador. Publicaciones, 50(2), 167-185.

Botero, C. (2009). Cinco tendencias de la gestión educativa. Revista Iberoamericana de Educación, 49(2), 1-11.

Cámara de Comercio de Quito [CCQ] (2021). Oferta Académica Escuela de Negocios Cámara de Comercio de Quito. Recuperado de https://www.ccq.edu.ec/Programas-Modalidad-Virtual

Castillo, J. y Powell, M. (2019). Análisis de la producción científica del Ecuador e impacto de la colaboración internacional en el periodo 2006-2015. Revista Española de Documentación Científica, 42(1), [s.p.].

Chiavenato, I. (2007). Introducción a la teoría general de la administración (7a ed.). México, Buenos Aires: McGraw-Hill Interamericana.

Chuaqui, B. (2002). Acerca de la historia de las universidades. Revista chilena de pediatría, 73(6), 563-565.

Consejo de Aseguramiento de la Calidad de la Educación Superior Ecuador [CACES] (2019). Modelo de evaluación externa de universidades y escuelas politéncias 2019.

Consejo de Educación Superior Ecuador [CES] (2020). Codificación Reglamento de Régimen Académico del Consejo de Educación Superior [RRA. CES]. Res. RPC-SO-08-No. 111-2019 y Resolución RPC-SO-16-No.3312020.

Consejo de Educación Superior Ecuador [CES] (2021). Reglamento de Carrera y Escalafón del Personal Académico del Sistema de Educación Superior, 2021. RPC-SE-19-No.055-2021.

Corte Constitucional del Ecuador [CCE] (2020). Sentencia No. 9-20-IA/20. Recuperado de http://esacc.corteconstitucional.gob.ec/storage/api/v1/10_DWL_FL/e2NhcnBIdGE6J3RyYW1pdGUnLCB1d WlkOicwYjc4ZTliMy03NjU5LTRiYWItYTI1Yy1jMzljM2M2MWM2NWUucGRmJ30=

Crozier, M. (1969). El fenómeno burocrático. Buenos Aires: Amorrortu.

Dáher, J., Panunzio, A., Hernández, M. (2018). La investigación científica: una función universitaria a considerar en el contexto ecuatoriano. Edumecentro 10(4) [s.p.]

Escobar, C. (2016). Análisis de la política ecuatoriana de becas de estudios de posgrado en el exterior y su relación con el cambio de matriz productiva*. Revista Latinoamericana de Políticas y Acción pública, 3(2), 23-49.

Fayol, H. (1987). Administración industrial y general (14a ed.). Buenos Aires: El Ateneo.

Garzón, M. (2019). Propuesta de modelo de universidad corporativa. Visión de Futuro, 23(1). [s.p.]

Hernández, R., Fernández, C. y Baptista, P. (2006). Metodología de la investigación (4a ed.). México, Buenos Aires: McGraw-Hill Interamericana.

Instituto Nacional de Estadísticas y Censos Ecuador [INEC] (2021). Encuesta Nacional de Empleo, Desempleo y Subempleo. Recuperado de https://www.ecuadorencifras.gob.ec/documentos/webinec/EMPLEO/2021/Trimestre-enero-marzo-2021/Trimestral\%20eneromarzo\%202021_Mercado_Laboral.pdf 
Jarvis, P. (2006). Universidades corporativas: Nuevos modelos de aprendizaje en la sociedad global. Madrid: Narcea.

Larraín, F. y Waissbluth, M. (2018). Modelos de gestión pública: implicancias para la planificación, evaluación y control de gestión del Estado. Un Mejor Estado Para Chile, 541-559.

López, R., Cedeño, G., Lescay, D., y Mestre, U. (2016). Relevancia del proceso de vinculación con la sociedad en la formación de profesionales en instituciones de educación superior del Ecuador. Órbita Pedagógica, 3(2), 61-82.

Merton, R. (1964). Estructura burocrática y personalidad. En Teoría y Estructura sociales [fuente reproducida] (p. 275-286). México: Fondo de Cultura Económica.

Mises, L. (2005). Burocracia: gestión empresarial frente a gestión burocrática (2a ed.). Madrid: Unión Editorial.

Mora, J. (2018). Universidades: mitos, modas y tendencias. Revista Iberoamericana de Educación Superior, 9(24), 3-16.

Muñoz, H. (2019). La burocracia universitaria. Revista de La Educación Superior, 48(189), 73-96.

Olsen, J. (2005). Quizás sea el momento de redescubrir la burocracia. Revista del CLAD Reforma y Democracia, $31,1-24$.

Ortega, A. y Guerrero, C. (2021). La política de Educación Superior en el Ecuador: Un ejercicio contextualizado de análisis crítico. Scientific, 6(20), 19-40.

Petrella, C. (2007). Análisis de la teoría burocrática: aportes para la comprensión del modelo burocrático. Revista electrónica de la Facultad de Ciencias Empresariales Universidad Católica del Uruguay, (2).

Román, M. (1976). Burocracia y cambio social [español ed.]. Madrid: J, Porrúa.

Rueda, I., Acosta, B., y Cueva, F. (2020). Las universidades y sus prácticas de vinculación con la sociedad. Educacao \& Sociedade, 41 [s.n., s.p.]

Saldaña, L. (2017). La Universidad como institución pertinente en el avance de la sociedad. Educación Médica Superior, 31(3), 222-231.

Secretaría de Educación Superior, Ciencia, Tecnología e Innovación Ecuador [SENESCYT] (2019). Estadísticas de educación superior, ciencia, tecnología e innovación: registro de matrícula de Universidades y Escuelas Politécnicas campo de conocimiento. Recuperado de https://siau.senescyt.gob.ec/estadisticas-deeducacion-superior-ciencia-tecnologia-einnovacion/?doing_wp_cron=1621265656.1037058830261230468750

Secretaría de Educación Superior, Ciencia, Tecnología e Innovación Ecuador [SENESCYT] (2020). Boletín anual: análisis anual de los principales indicadores de educación superior, ciencia, tecnología e innovación. Recuperado de https://www.educacionsuperior.gob.ec/wpcontent/uploads/2020/09/Boletin_Anual_Educacion_Superior_Ciencia_Tecnologia_Innovacion_Agosto20 20.pdf

Secretaría de Educación Superior, Ciencia, Tecnología e Innovación Ecuador [SENESCYT] (2020). Innovacyt capital semilla para emprendedores. Recuperado de http://www.bancodeideas.gob.ec/ 
Secretaría Nacional de Planificación y Desarrollo Ecuador [SENPLADES] (2012). Folleto Informativo Transformación de la Matriz Productiva. Recuperado de https://www.planificacion.gob.ec/wpcontent/uploads/downloads/2013/01/matriz_productiva_WEBtodo.pdf

Strauss, A. y Corbin, J. (2002). Bases de la investigación cualitativa. Técnicas y procedimientos para desarrollar la teoría fundamentada (español ed.) Antioquia: Universidad de Antioquia.

Taylor, F. (1987). Principios de la administración científica (10a ed.). Buenos Aires: El Ateneo.

Torres, Z. (2014). Teoría general de la Administración (2a ed.). México: Patria.

Universidad Técnica de Ambato [UTA] (2020). Resolución 1222-CU-P-2020, 2020. Recuperado de https://redi.uta.edu.ec/handle/123456789/79981

Viña, N., Sacoto, V., y Landívar, J. (2019). Contribución de la Universidad ecuatoriana en beneficio de sectores vulnerables - Vinculación con la Sociedad. Espacios. 40(23), 1-11. Recuperado de:

http://www.revistaespacios.com/a19v40n23/a19v40n23p11.pdf

Vizioli, E. (2016) La innovación en la política pública de ciencia y tecnología ecuatoriana. Yachay: ¿Un cluster de innovación?. (Tesis de Maestría). Facultad Latinoamericana de Ciencias Sociales sede Ecuador [FLACSO], Quito.

Weber, M. (2001). ¿Qué es la burocracia? (español ed.). México: Coyoacán.

Zamudio, L. y Arellano, D. (2011). Más allá de las patologías de la burocracia: introduciendo la teoría de las organizaciones al estudio de las OI. Revista Mexicana de Ciencias Políticas y Sociales, 56(213), 27-50.

Esta obra está bajo una Licencia Creative Commons Attribución-NoCommercial 4.0 International

\section{(cc) EYY-NC}

